\title{
Comparison of Necroptosis With Apoptosis for OVX-Induced Osteoporosis
}

\author{
Bin $\mathrm{He}^{1,2+}$, Yongjun Zhu ${ }^{3+}$, Hongwang Cui ${ }^{1 *}$, Bo Sun ${ }^{1}$, Tian Su ${ }^{1}$ and Peng Wen ${ }^{1}$ \\ ${ }^{1}$ Department of Spine and Osteopathic Surgery, The First Affiliated Hospital of Hainan Medical University, Haikou, China, \\ ${ }^{2}$ Department of Orthopedics, The First Affiliated Hospital of Chongqing Medical University, Chongqing, China, ${ }^{3}$ Department of \\ Nephrology, The First Affiliated Hospital of Hainan Medical University, Haikou, China
}

OPEN ACCESS

Edited by:

Paulo Sergio Cerri,

Universidade Estadual Paulista, Brazil

Reviewed by:

Victor E. Arana-Chavez,

University of São Paulo, Brazil

Rafael Pacheco Da Costa, University of Western São Paulo, Brazil

Rinaldo Florencio-Silva, Universidade Federal de Sao Paulo,

Brazil

*Correspondence: Hongwang $\mathrm{Cu}$

cqchw2013@sina.com

${ }^{\dagger}$ These authors have contributed equally to this work

Specialty section: This article was submitted to Cellular Biochemistry,

a section of the journal

Frontiers in Molecular Biosciences

Received: 07 October 2021 Accepted: 29 November 2021

Published: 24 December 2021

Citation:

He B, Zhu Y, Cui H, Sun B, Su T and

Wen $P$ (2021) Comparison of Necroptosis With Apoptosis for OVXInduced Osteoporosis.

Front. Mol. Biosci. 8:790613. doi: 10.3389/fmolb.2021.790613
As one common kind of osteoporosis, postmenopausal osteoporosis (PMOP) is associated with the death and excessive loss of osteocytes. Estrogen deficiency of PMOP can cause osteocyte death by regulating necroptosis and apoptosis, but their roles in POMP have not been compared. In the present study, ovariectomy (OVX)-induced rat and murine long bone osteocyte Y4 (MLO-Y4) cells were used to compare the influence of necroptosis and apoptosis on osteocyte death and bone loss. Benzyloxycarbonyl-ValAla-Asp (zVAD) and necrostatin-1 (Nec-1) were used to specifically block cell apoptosis and necroptosis, respectively. OVX rats and MLO-Y4 cells were divided into ZVAD group, Nec-1 group, zVAD + Nec-1 group, vehicle, and control group. The tibial plateaus of the rat model were harvested at 8 weeks after OVX and were analyzed by micro-computed tomography, transmission electron microscopy (TEM), the transferase dUTP nick end labeling assay, and western blot. The death of MLO-Y4 was stimulated by TNF- $\alpha$ and was measured by flow cytometry and TEM. The results found that necroptosis and apoptosis were both responsible for the death and excessive loss of osteocytes, as well as bone loss in OVX-induced osteoporosis, and furthermore necroptosis may generate greater impact on the death of osteocytes than apoptosis. Necroptotic death of osteocytes was mainly regulated by the receptor-interacting protein kinase 3 signaling pathway. Collectively, inhibition of necroptosis may produce better efficacy in reducing osteocyte loss than that of apoptosis, and combined blockade of necroptosis and apoptosis provide new insights into preventing and treating PMOP.

Keywords: necroptosis, apoptosis, ovariectomy, RIPK3, in vivo, in vitro, osteocytes

\section{INTRODUCTION}

Osteoporosis is one systemic skeletal disease characterized by low bone mass and microarchitectural deterioration of bone tissue, which increases susceptibility to fractures (Ensrud and Crandall, 2017; Kanis et al., 2019). With the aging of a population, the incidence of postmenopausal osteoporosis (PMOP) is enormously increased and may lead to serious osteoporotic fractures (Fang et al., 2020; He et al., 2021; Zhao et al., 2021). Osteocytes, osteoclasts, and osteoblasts have important roles in bone remodeling and the incidence of osteoporosis (Han et al., 2018; Robling and Bonewald, 2020; Udagawa et al., 2021). Osteocytes are known as ramified bone cells distributed throughout the bone matrix and subsequently form 90-95\% of adult bone cells (Milovanovic et al., 2013; Mattinzoli et al., 2014). Osteocytes play a 
critical role in transmitting various signals to the surfaces between osteoclasts and osteoblasts (Kalajzic et al., 2013), suggesting that osteocytes may also participate in regulating bone resorption and formation (Heino et al., 2004). Especially, osteocyte death is confirmed to cause PMOP (Dallas et al., 2013).

Increasing evidence indicates that ongoing loss of osteocytes caused by cell death is an important factor that results in PMOP, and osteocyte apoptosis has been reported to be a crucial factor (Cui et al., 2016a; Vakili et al., 2021). Previous studies have demonstrated that apoptosis of osteocytes is accelerated within 4 weeks of estrogen withdrawal (Cardoso et al., 2009; Emerton et al., 2010). In addition to apoptosis, necroptosis is another important approach that causes cell death and is regarded as one subtype of necrosis (Degterev et al., 2014). Necrotic cell death modalities contribute to different pathologies including necroptosis, pyroptosis, and parthanatos (Robinson et al., 2019). Apoptosis is programed cell death with the features of cell shrinkage and nuclear condensation and is regulated by multiple pathways including the autophagy pathway, mitogen-activated protein kinase-dependent antioxidant signaling, overproduction of reactive oxygen species, and WNT/ $\beta$-catenin signaling (Ru and Wang, 2020). Necroptosis is highly regulated caspase-independent programed cell death with morphological similarities to necrosis, including increased cell volume, organelle swelling, plasma membrane rupture, and subsequent loss of intracellular contents, which are regulated by receptorinteracting protein kinase 1/3 (RIPK1/RIPK3) signaling and can be blocked by necrostatin-1 (Nec-1) intervention (Linkermann et al., 2012; Cui et al., 2016a). Many inflammatory signals such as tumor necrosis factor-a (TNFa) and Toll-like receptors also participate in the pathological processes of necroptosis (Belizário et al., 2015).

Apoptosis and necroptosis, two different types of cell death, display crucial roles in the loss of osteocyte and bone mass. Estrogen loss in ovariectomy (OVX) animals significantly increases apoptosis and necroptosis of osteocytes, as well as osteoclastic resorption (Emerton et al., 2010; Cui et al., 2016b), which are modulated by many factors including the types of bone tissues, specific compartments, and the time of OVX (Emerton et al., 2010; Gerbaix et al., 2017; Fu et al., 2020). Our previous studies demonstrated that necroptosis is one important approach to cause osteocyte death and subsequent osteoporosis in rats undergoing OVX (Cui et al., 2016a; Cui et al., 2016b). The progressive depletion of osteocytes due to necroptosis promoted the progression of PMOP in OVX rats, and necroptotic death of osteocytes could be blocked by treatment with necrostatin-1 (Nec-1, a specific inhibitor of receptorinteracting protein 1), which subsequently attenuated bone loss (Cui et al., 2016b). However, the roles of necroptosis and apoptosis for osteocyte loss have not been well established, and the aim of the present study is to compare necroptosis with apoptosis in the progressive loss of osteocytes in rats with estrogen deficiency.

\section{MATERIALS AND METHODS}

\section{Animals and Grouping}

The study protocol was approved by the ethics committee of the First Affiliated Hospital of Hainan Medical University (Haikou, China). All animal experimental procedures were carried out in accordance with the guidelines laid down by the National Institute of Health (United States) and the National Laws and Regulations.

Female Sprague Dawley (SD) rats aged 16 weeks (Animal Laboratory Center of Chongqing Medical University, Chongqing, China) were housed under standard laboratory conditions (12-h light/dark cycle, temperature $22 \pm 2^{\circ} \mathrm{C}$, and humidity $55 \pm 5 \%$ ) with free access to water and standard rodent diet for a 7-day adaptation period. A total of 62 animals were randomly divided into OVX group (bilateral OVX, $\mathrm{n}=50$ ) and control group (sham operation, $\mathrm{n}=12$ ). Anesthesia was administered by subperitoneal injection with pentobarbital sodium $(30-50 \mathrm{mg} / \mathrm{kg})$. Among the OVX group, two rats died during the bilateral OVX surgery because of deep anesthesia. For the sham operation in the control group, the ovaries were exteriorized and then replaced back in the abdominal cavity. We observed the postoperative recovery, diet, wound healing, and daily activities after OVX. All rats were housed under standard laboratory conditions with free access to water and a standard rodent diet. All of them gradually obtained complete recovery and good wound healing.

\section{Drug Administration}

Drugs included necrostatin-1 (Nec-1, Sigma-Aldrich, United States) and benzyloxycarbonyl-Val-Ala-Asp (zVAD, MP Biomedicals, Solon, OH, United States), which were dissolved in $10 \%$ dimethyl sulfoxide (DMSO, Sigma, United States) for subsequent use. zVAD and Nec-1 were used to specifically block cell apoptosis and necroptosis, respectively (Cui et al., 2016b; Ormsby et al., 2019). After 4 weeks of recovery after OVX, 48 rats in the OVX group were randomly assigned into four subgroups: OVX + vehicle group, OVX $+\mathrm{zVAD}$ group, $\mathrm{OVX}+\mathrm{Nec}-1$ group, and OVX + zVAD + Nec-1 group. Nec-1 $(1.65 \mathrm{mg} / \mathrm{kg} / \mathrm{d})$ (Linkermann et al., 2012), zVAD (1.0 mg/kg/d) (Baumann et al., 2009), or an equal volume of $10 \%$ DMSO as the vehicle was injected intraperitoneally into the rats once per day for 4 weeks in the corresponding groups. The animals in the control group were administered with the vehicle solution. The rats were sacrificed 8 weeks after the operation by cervical dislocation because the peak time of osteocyte death was reported to be at 8 weeks after OVX (Cui et al., 2016a).

\section{Sample Harvest}

Both the right and left tibial plateaus were dissected, and the soft tissue was removed from the bone. The bone marrow was rinsed out from the tibial plateau by repeated washing with PBS. Only the bone matrix of the tibial plateaus were stored in liquid nitrogen for subsequent western blot analysis $(n=6$ rats per group). Some tibial plateaus were fixed in $10 \%$ neutral buffered formalin for $24 \mathrm{~h}$ for micro-computed tomography (micro-CT) analysis and histological analysis. The tibial plateaus used for the 
histological analysis were decalcified in $15 \%$ ethylenediaminetetraacetic acid (EDTA, $\mathrm{pH}$ 7.4) for 4 weeks and then subjected to the transferase dUTP nick end labeling (TUNEL) and immunohistochemistry reaction for detection of caspase-3. Other tibial plateaus were fixed in $2.5 \%$ glutaraldehyde for $24 \mathrm{~h}$ at $4^{\circ} \mathrm{C}$ and decalcified in 15\% EDTA (pH 7.4) for 4 weeks for transmission electron microscopy (TEM).

\section{Micro-Computed Tomography}

To analyze the bone microarchitecture, the tibial plateaus were scanned with micro-CT (viva CT40, SCANCO Medical AG, Zürich, Switzerland) using the following conditions: $15 \mu \mathrm{m}$ resolution, $70 \mathrm{kVp}, 114 \mu \mathrm{A}$, and $250 \mathrm{~ms}$ integration time. The volume of interest $\left(29 \times 29 \times 29 \mu^{3}\right)$ was selected using a semiautomatic contouring method. 3D images were constructed using a series of planar transverse gray-value images. The microarchitectural parameters including bone mineral density (BMD), bone volume fraction (BV/TV), trabecular thickness (Tb.Th), trabecular number (Tb.N), and trabecular separation were calculated automatically using the micro-CT software.

\section{Western Blotting}

The tibial plateaus frozen in liquid nitrogen were pulverized and homogenized in ice-cold buffer (Beyotime, Nantong, Jiangsu, China). The total protein concentration was determined with the bicinchoninic acid protein assay kit (Beyotime, Nantong, Jiangsu, China) according to the operating manual. The protein levels were determined by measuring the absorbance at $280 \mathrm{~nm}$. Approximately $80 \mu \mathrm{g}$ of protein was separated by SDS-PAGE and transferred to the polyvinylidene difluoride membrane (EMD Millipore, United States), which was then blocked with $5 \%$ nonfat dry milk solution for $1.5 \mathrm{~h}$ at room temperature and incubated overnight at $4^{\circ} \mathrm{C}$ with the following primary antibodies: anti-RIPK3 polyclonal antibody (ab152130, Abcam, Inc., Cambridge, MA, United States, 1:1000 dilution), anti-cleaved caspase-3 polyclonal antibody (ab2302, Abcam, Inc., Cambridge, MA, United States, 1:1000 dilution), anti-TNF- $\alpha$ monoclonal antibody (ab205587, Abcam, Cambridge, MA, United States, 1:1000 dilution), or anti- $\beta$-actin monoclonal antibody (sc-47778, Santa Cruz Biotechnology, CA, United States, 1:1000 dilution). Western blotting was performed routinely as previously described (Cui et al., 2016b). The protein bands of interest were visualized using an external cavity laser detection kit (Beyotime, Nantong, Jiangsu, China). The protein expression was quantitated by densitometry using Image Lab version 2.1 (Bio-Rad) and quantitative densitometric values for each kind of protein were normalized to $\beta$-actin.

\section{Immunofluorescence and In Situ Fluorescence TUNEL Staining}

The bone matrix of the tibial plateaus was sliced into $4-\mu \mathrm{m}$ sections for immunofluorescence and in situ fluorescence TUNEL staining. After three washes with $0.1 \mathrm{M}$ PBS, the sections were incubated with $20 \mu \mathrm{g} / \mathrm{ml}$ proteinase $\mathrm{K}$ at $37^{\circ} \mathrm{C}$ for $15 \mathrm{~min}$, treated with $0.1 \%$ Triton $\mathrm{X}-100$, blocked with $10 \%$ goat serum, and incubated with polyclonal antibody against cleaved caspase-3 (Cell Signaling Technologies, Danvers, MA, United States; 1:100 dilution) overnight at $4^{\circ} \mathrm{C}$, followed by anti-mouse IgG (EarthOx, San Francisco, United States). After three washes with PBS, the sections were analyzed by using the in situ cell death detection kit (Roche, Basel, Switzerland) following the manufacturer's protocol for the TUNEL method. All the sections were counterstained with $4^{\prime}, 6$ diamidino-2-phenylindole (DAPI). Finally, the number of total cells and TUNEL-positive cells with immunostaining after caspase-3 (with and without cleaved caspase-3 immunostaining) were counted in three to five noncontiguous high-power fields $(\times 200$ magnification) for each specimen under a laser scanning confocal microscope (Leica TCS SP2, Wetzlar, Germany). The percentage and number of TUNEL-positive and cleaved caspase-3-positive cells were calculated by an experienced pathologist who was blinded to the experimental conditions.

\section{Transmission Electron Microscopy}

The tibial plateau $\left(1 \mathrm{~mm}^{3}\right)$ was fixed in $2.5 \%$ glutaraldehyde for

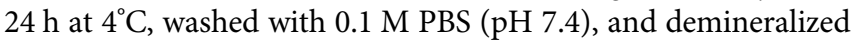
in $15 \%$ EDTA for 4 weeks at room temperature. After three washes with PBS, tissue fragments were postfixed in $2 \%$ osmium tetroxide for $1 \mathrm{~h}$, block-stained with $2 \%$ uranyl acetate, embedded in epoxy resin, and sliced into $80-\mathrm{nm}$ sections which were subsequently stained with uranyl acetate and lead citrate and finally observed for the ultrastructures by using a transmission electron microscope (Hitachi-7500, Hitachi, Tokyo, Japan).

\section{Cell Culture and Treatments}

Murine long bone osteocyte Y4 (MLO-Y4, 2.5×10 4 cells, Cell Bank of the Chinese Academy of Sciences, Beijing, China) were plated in 12-well plates and cultured in DMEM/F12 (Gibco Life Technologies, Carlsbad, CA, United States) supplemented with $10 \%$ fetal bovine serum (FBS; Gibco Life Technologies, Carlsbad, $\mathrm{CA}$, United States) in a humidified incubator containing $5 \% \mathrm{CO}_{2}$ at $37^{\circ} \mathrm{C}$. The cells were pretreated with DMSO (1\%), Nec-1 (30 mmol/L), zVAD (25 mmol/L), or Nec-1 (30 mmol/L) + $\mathrm{zVAD}(25 \mathrm{mmol} / \mathrm{L})$ for $30 \mathrm{~min}$ at $37^{\circ} \mathrm{C}$, and then treated with TNF-a (100 ng/ml) for $24 \mathrm{~h}$ (Zhu et al., 2016). Flow cytometric analysis was performed using a FITC Annexin V apoptosis detection kit (BD Biosciences, San Diego, CA) according to the manufacturer's protocol. The cells were harvested, washed twice with PBS, and resuspended in $100 \mu \mathrm{L}$ binding buffer. Cells were incubated for $15 \mathrm{~min}$ at room temperature in the dark after adding $5 \mu \mathrm{L}$ FITC Annexin V and propidium iodide (PI), and then diluted with $400 \mu \mathrm{L}$ of binding buffer. The cells were analyzed by flow cytometry (BD Biosciences, San Jose, CA). The cells in the four different quadrants were analyzed and interpreted as Q1: necrosis (Annexin V-/PI+), Q2: late apoptosis (Annexin $\mathrm{V}+/ \mathrm{PI}+$ ), Q3: early apoptosis (Annexin V+/PI-), and Q4: viable cells (Annexin V-/PI-) (Malik et al., 2020). The percentages of late apoptosis plus necrosis and early apoptosis were calculated. Then, the cells $\left(5 \times 10^{6}\right)$ were harvested and prefixed with $2.5 \%$ glutaraldehyde for $2 \mathrm{~h}$. These cells were observed by TEM as described previously. The percentage of dead cells was calculated in six noncontiguous fields $(\times 6,000$ magnification) in each group. 


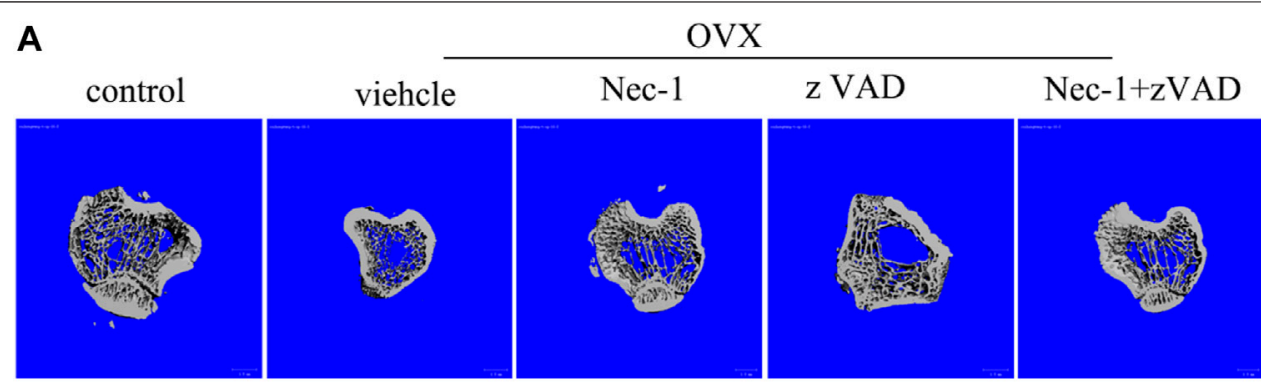

B

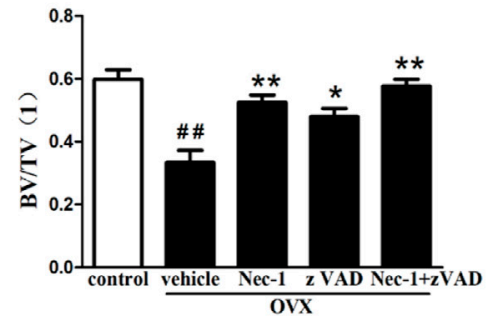

D

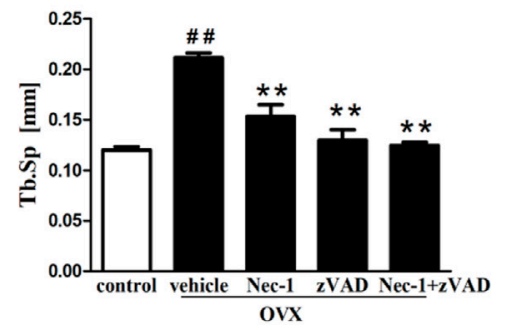

C

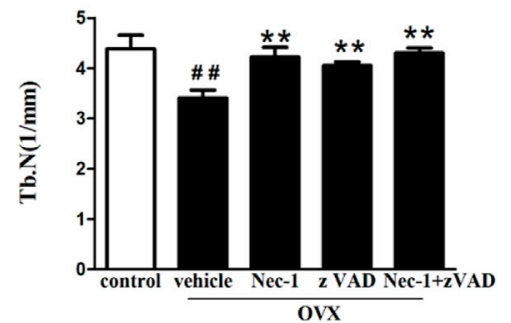

E

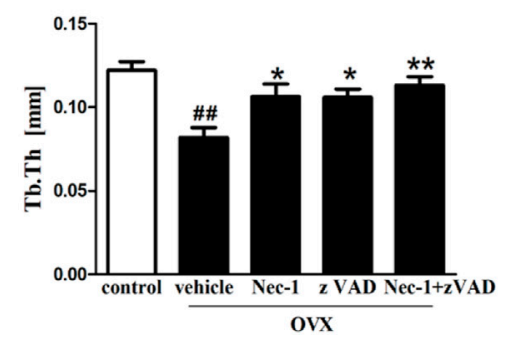

FIGURE 1 | Bone loss was measured by micro-CT in OVX rats. The bone matrix of the tibial plateau was scanned using micro-CT, and the microarchitectural parameters (BV/TV, Tb.Th, Tb.Sp, and Tb.N) were calculated automatically. (A) Representative 2D reconstructed images were obtained in each group (scale bar = $100 \mu \mathrm{m})$ (B) BV/TV, (C) Tb.Th, (D) Tb.Sp, and (E) Tb.N were compared ( $\mathrm{n}=4)$. \#\#p < 0.01 compared with the control group, ${ }^{*} p<0.05$ compared with the vehicle group, ${ }^{\star \star} p<0.01$ compared with the vehicle group.

\section{Statistical Analysis}

Data were presented as mean \pm standard deviation (SD). Statistical analysis was performed using SPSS software version 22.0. The significant differences between groups were evaluated using ANOVA followed by the least significant difference or Dunnett's post hoc test. The $p$ value of $<0.05$ was considered statistically significant.

\section{RESULTS}

\section{Bone Loss in OVX Rats}

About 8 weeks after OVX in SD rats, bone microarchitectures were measured by micro-CT (Figure 1). Figure 1A demonstrates the $2 \mathrm{D}$ reconstruction of the tibial plateaus and the BV/TV (Figure 1B), Tb.N (Figure 1C), Tb.Sp (Figure 1D), and Tb.Th (Figure 1E) in each group were compared. Compared to that in the control group, OVX led to significantly decreased BV/TV (Figure 1B), Tb.N (Figure 1C), and Tb.Th (Figure 1E) and increased Tb.Sp (Figure 1D), confirming that OVX resulted in substantial loss of bone mass and osteoporosis $(p<0.05)$.
Compared with the vehicle group, BV/TV (Figure 1B), Tb.N (Figure 1C), and Tb.Th (Figure 1E) were remarkably increased in the Nec-1 group, zVAD group, and Nec-1 + zVAD group $(p<$ 0.05 ), accompanied by reduced Tb.Sp (Figure 1D). In terms of $\mathrm{BV} / \mathrm{TV}$, the Nec-1 group, zVAD group, and Nec-1 + zVAD group led to approximately $57 \%$ increase, $44 \%$ increase, and $73 \%$ increase than vehicle group, respectively (Figure 1B). These three groups were also associated with about $24 \%$ increase, $19 \%$ increase, and $26 \%$ increase in Tb.N relative to the vehicle group (Figure 1C). For Tb.Sp, we found approximately $27 \%$ decrease in Nec-1 group, 39\% decrease in zVAD group, and $41 \%$ decrease in the Nec-1 + zVAD group compared to the vehicle group (Figure 1D). Regarding Tb.Th, these three groups results in about 30\% increase, $29 \%$ increase, and $40 \%$ increase than vehicle group (Figure 1E). These results suggested that treatment with Nec-1, zVAD and especially Nec-1 + zVAD could improve bone mass as compared to the vehicle group.

\section{Dead Osteocytes by TUNEL Staining}

DNA fragments produced by dead cells in the bone matrix of the tibial plateau were detected by the TUNEL assay 


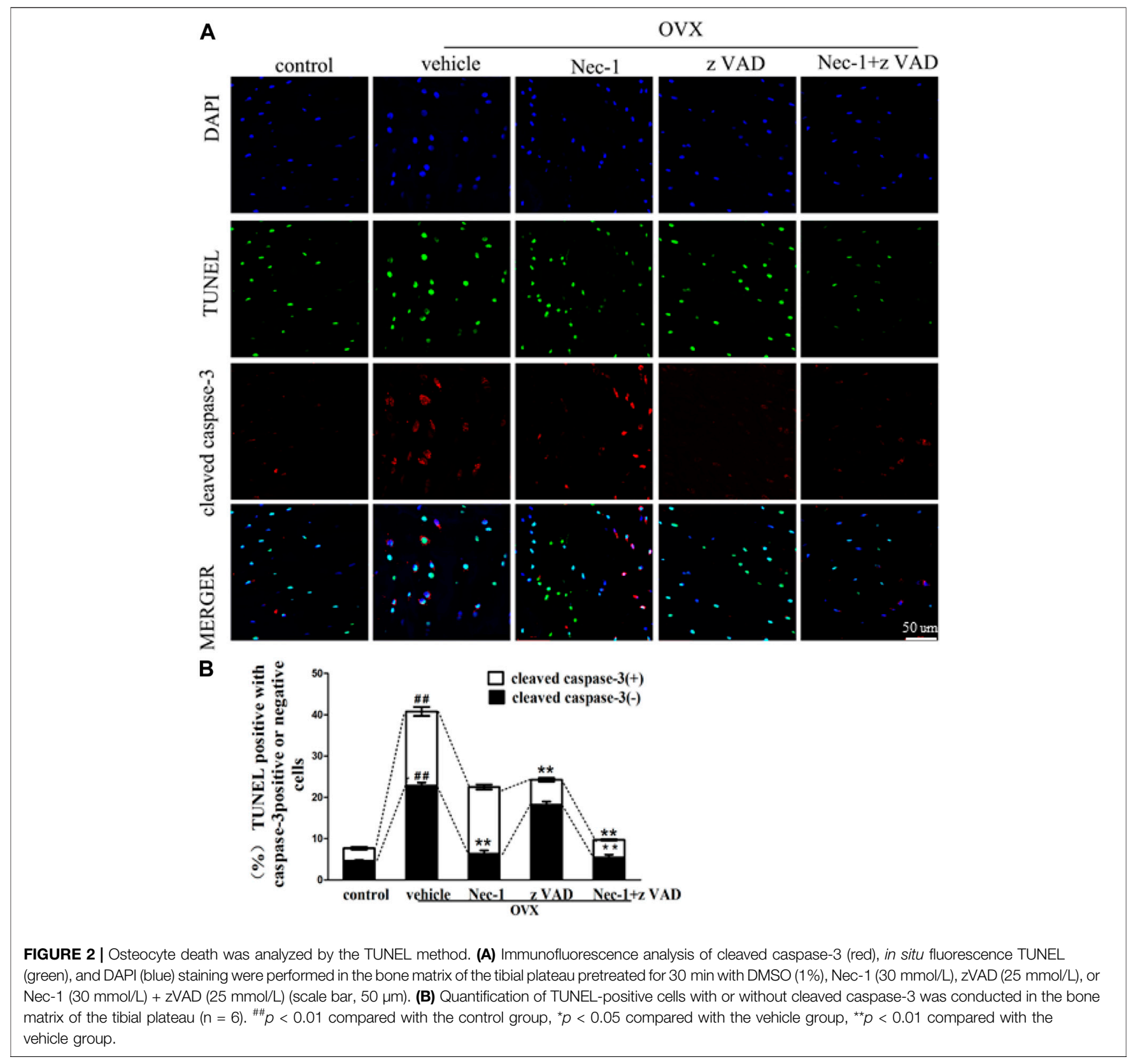

(Figure 2A). The percentage of TUNEL-positive cells in the bone matrix of the tibial plateau in the vehicle group was greater than in the control group (Figure 2B). The percentage of TUNEL-positive cells was reduced significantly after treatment with $\mathrm{zVAD}$ or Nec-1 alone, and the significance of reduction became more robust in the $\mathrm{Nec}-1+\mathrm{zVAD}$ group. It was important to emphasize that the number of TUNELpositive cells in OVX rats treated with $\mathrm{Nec}-1$ or $\mathrm{Nec}-1+\mathrm{zVAD}$ was significantly lower than in rats treated with $\mathrm{zVAD}$ alone (Figures 2A,B). Furthermore, anti-active caspase-3 staining was conducted to identify cell apoptosis (Günther et al., 2011; Liedtke et al., 2011; Vanlangenakker et al., 2012). These results indicated that both necroptosis and apoptosis led to the death and loss of osteocytes, and cell necroptosis had a bigger role in the loss of osteocytes than cell apoptosis.

\section{Morphological Observation of Osteocytes by TEM}

To further examine the morphological characteristics of dead osteocytes, we observed the ultrastructures of osteocytes in the bone matrix of the tibial plateaus using TEM at 8 weeks after OVX. The typical morphological features of necroptotic osteocytes included swelling of the organelles, rupture of the plasma membrane, 


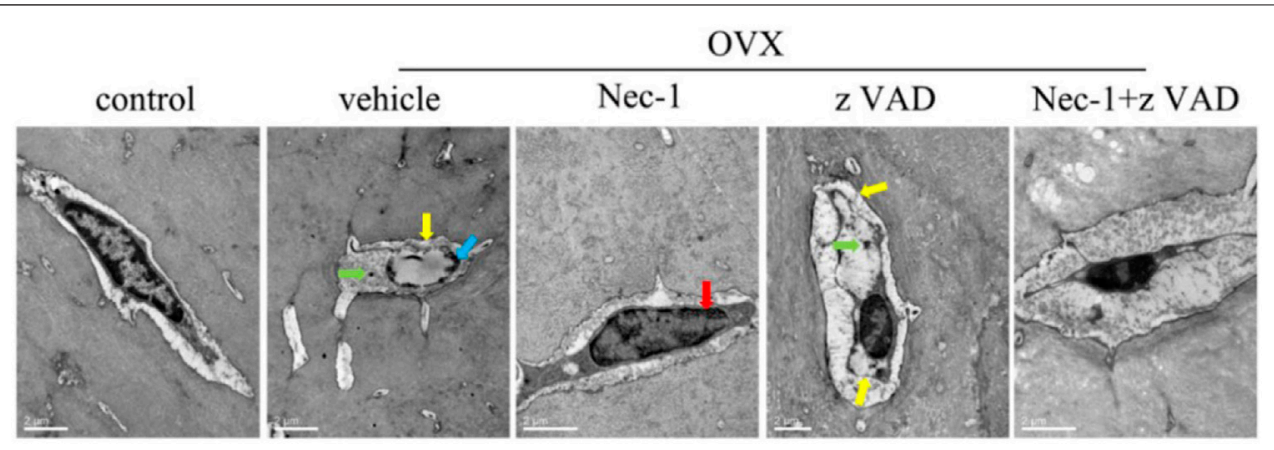

FIGURE 3|Dead osteocytes were seen by TEM. (A) TEM images of osteocytes in sham-operated rats and OVX rats treated with vehicle, Nec-1, zVAD, or Nec-1 + zVAD (scale bar $=2 \mu \mathrm{m})$. Red arrow: condensed nucleus, blue arrow: chromatin clumping, green arrow: swelling organelle, yellow arrow: membrane rupture. \#\#p < 0.01 compared with the control group, ${ }^{*} p<0.05$ compared with the vehicle group, ${ }^{\star \star} p<0.01$ compared with the vehicle group.

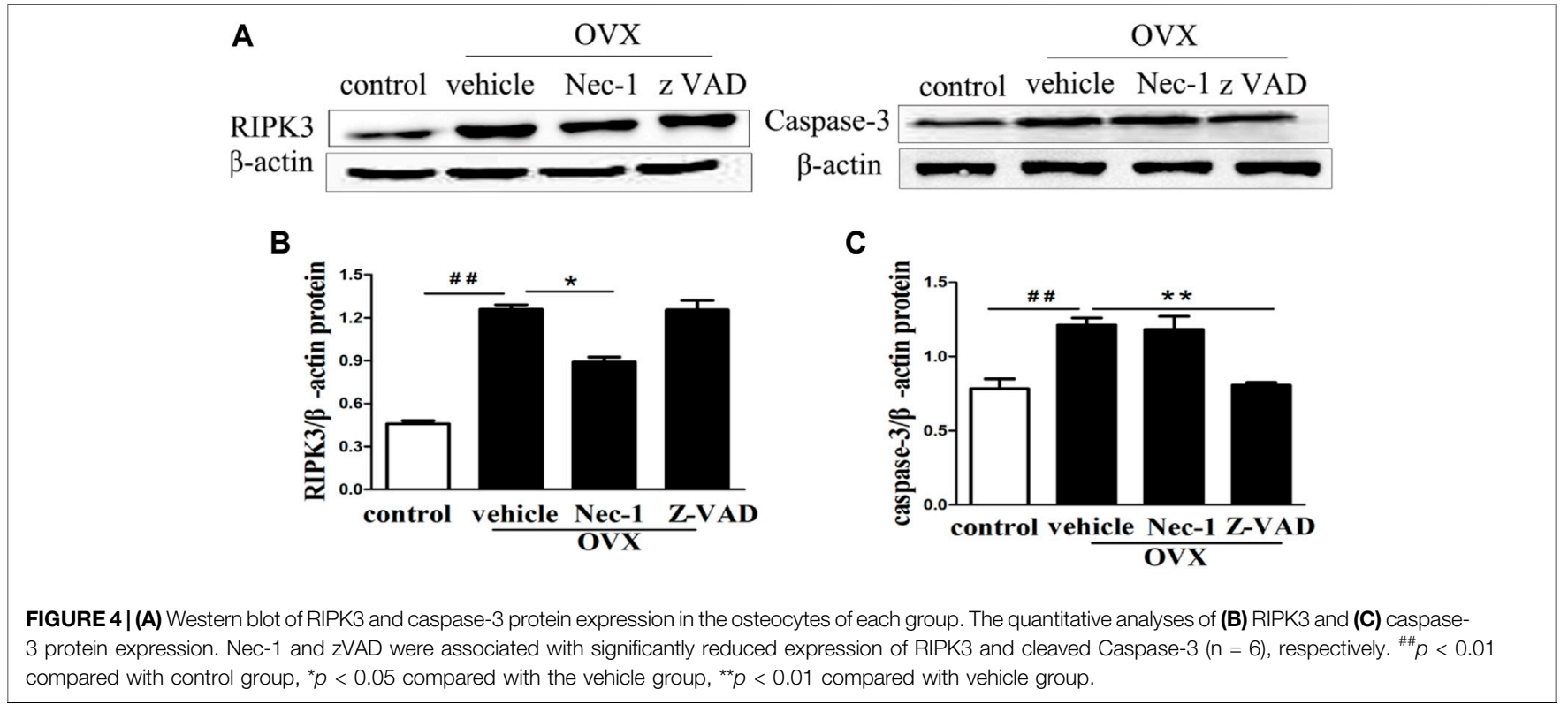

chromatin clumping, and intracellular vacuoles, which were seen in the vehicle group and $\mathrm{zVAD}$ group. Nuclear condensation of apoptotic cells was observed in the Nec-1 group (Figure 3). These indicated that osteocyte death was mainly caused by necroptosis rather than apoptosis at 8 weeks after OVX.

\section{RIPK3 and Caspase-3 Expression}

RIPK3 and cleaved caspase-3 were the important markers to identify necroptosis and apoptosis of cells, respectively. Their protein expression levels were measured in the bone matrix of OVX rats by using western blotting. The results showed that RIPK 3 and caspase- 3 protein levels were increased in the bone matrix of OVX rats compared with those in sham-operation rats (Figure 4A). Treatment with $\mathrm{Nec}-1$ significantly reduced the RIPK3 level (Figure 4B), while the caspase-3 level was substantially decreased in the zVAD group than in the Nec-1 group (Figure 4C). This confirmed that both necroptosis and apoptosis contributed to the death and loss of osteocytes.

\section{TNF- $\alpha$ Production in the Bone Marrow of OVX Rats}

TNF- $\alpha$ is mainly produced in hematopoietic precursor cells and is one important inflammatory factor which induces PMOP (Liu et al., 2020; Lu et al., 2020). TNF- $\alpha$ could directly enhance the expression of the receptor activator of nuclear factor $\kappa \mathrm{B}$ ligand (RANKL) in osteocytes and promote osteoclast formation (Kitaura et al., 2020). We detected the expression of TNF- $\alpha$ in the bone marrow of OVX rats in each group by western blotting and found that TNF- $\alpha$ expression was significantly lower in the control group and increased sharply in the vehicle group (Figures 5A,B). Although treatment with zVAD or Nec1 showed a decreasing trend in TNF- $\alpha$ protein expression compared to the vehicle group, no statistical difference was seen between them.

\section{TNF- $\alpha-$ Induced Death of MLO-Y4 Cells}

Estrogen deficiency increased the expression of TNF- $\alpha$ in the bone marrow of OVX rats, and we explored the influence of TNF- 

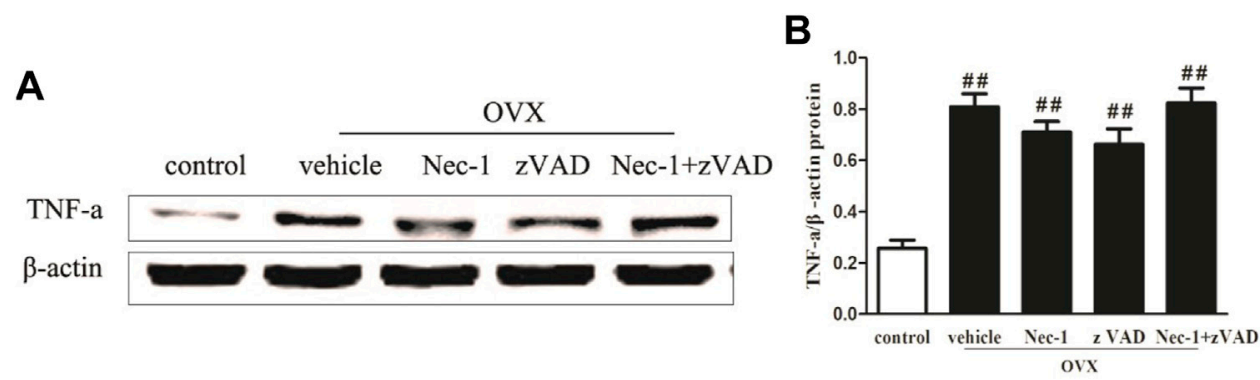

FIGURE 5 | TNF- $\alpha$ expression in OVX rats. (A) Western blot of TNF- $\alpha$ protein expression and (B) its quantitative analysis in the osteocytes of sham-operated and $\mathrm{OVX}$ rats treated with vehicle, Nec-1, or ZVAD $(n=6)$. \#\#p $<0.01$ compared with the control group, ${ }^{*} p<0.05$ compared with the vehicle group, ${ }^{* *} p<0.01$ compared with the vehicle group.
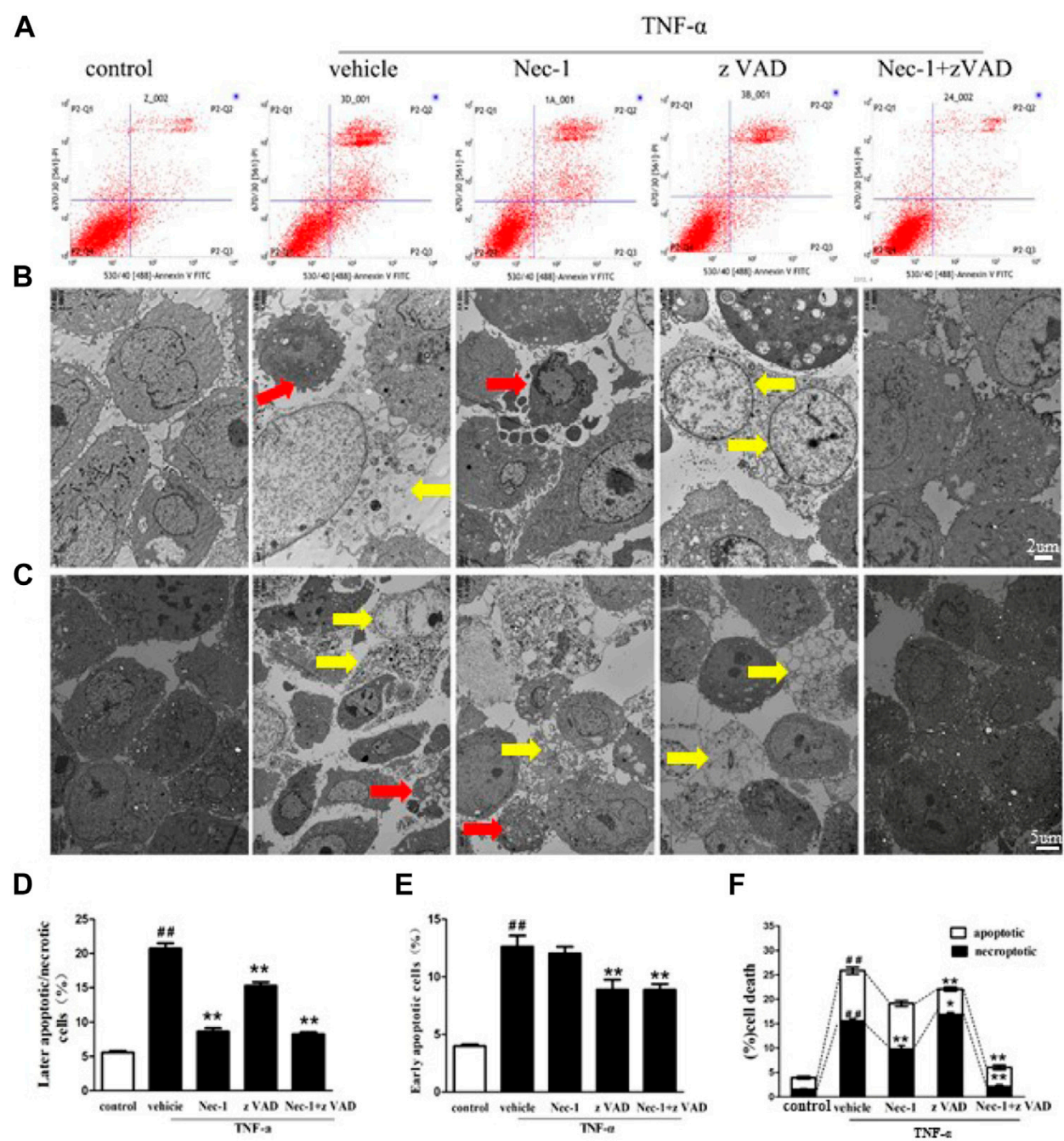

FIGURE 6 | TNF- $\alpha$-induced necroptosis and apoptosis of MLO-Y4 cells. (A, D, E) Necrotic and apoptotic osteocytes were tested using the flow cytometer. MLOY4 cells were pretreated for 30 min with DMSO (1\%), Nec-1 (30 mmol/L), zVAD (25 mmo//L), or Nec-1 (30 mmo//L) + zVAD (25 mmol/L) and then treated with TNF- $\alpha$ (100 ng/ml) for $24 \mathrm{~h}$ in vitro. Then, MLO-Y4 (upper panel) cells were stained with FITC Annexin V and PI to determine necrotic and apoptotic osteocytes by flow cytometry. (B, C, F) TEM images of osteocytes pretreated for 30 min with DMSO (1\%), Nec-1 (30 mmol/L), zVAD (25 mmol/L), or Nec-1 (30 mmol/L) + zVAD $(25 \mathrm{mmol} / \mathrm{L})$ and then treated with TNF- $\alpha(100 \mathrm{ng} / \mathrm{ml})$ for $24 \mathrm{~h}$ in vitro (B: scale bar $=2 \mu \mathrm{m}$, C: scale bar $=5 \mu \mathrm{m})$. Red arrow: apoptotic cells, yellow arrow: necroptotic cells. \#\#p 0.01 compared with the control group, ${ }^{\star} p<0.05$ compared with the vehicle group, ${ }^{\star \star} p<0.01$ compared with the vehicle group. 
a abnormality on the death of osteocytes. Therefore, we cultured MLO-Y4 cells in order to compare necroptosis with apoptosis for osteocyte death. Flow cytometry and TEM analyses revealed that the cell death rate of MLO-Y4 cells stimulated by TNF- $\alpha$ was significantly increased. Compared with the control group, early rate of cell death in the TNF- $\alpha$ group was significantly increased, and zVAD effectively reduced the early death rate of MLO-Y4 cells, but Nec-1 had no significant effect on the early death rate (Figures 6A,D). The late death rate in the TNF- $\alpha$ group was also significantly higher than in the control group. Both Nec-1 and $\mathrm{zVAD}$ remarkably reduced late necrosis and apoptosis of MLOY 4 cells, and Nec-1 could result in a significantly lower rate of cell death than did zVAD (Figures 6A,E). By contrast, the incidence of necroptotic cells was greater than that of apoptotic MLO-Y4 cells in response to TNF- $\alpha$ stimulation (Figure 6B, C, F). TNF- $\alpha$ combined with $\mathrm{zVAD}$ significantly decreased the rate of apoptosis but increased the incidence of necroptosis. Furthermore, TNF- $\alpha$ combined with $\mathrm{Nec}-1$ only blocked necroptosis but did not change the rate of apoptosis (Figure 6B, C, F). In addition, Nec-1 plus zVAD sharply reduced the incidence of apoptotic and necroptotic MLO-Y4 cells compared to vehicle intervention. These results confirmed that the death of osteocytes after TNF- $\alpha$ stimulus was caused by necroptosis and apoptosis, and cell necroptosis had a bigger role in the loss of osteocytes than did cell apoptosis. These findings are consistent with the results in vivo.

\section{DISCUSSION}

Death and loss of osteocytes display crucial roles in the incidence of PMOP, and osteocyte apoptosis has been widely accepted as an important pathological process in PMOP (Tomkinson et al., 1998; Emerton et al., 2010). Surprisingly, our previous study found that osteocyte necroptosis was also responsible for osteocyte loss in OVXinduced osteoporosis (Cui et al., 2016a). Both apoptotic and necroptotic cell deaths are regarded as two important approaches to cause excessive loss of osteocytes (Cui et al., 2016a), but their roles in the loss of osteocytes and PMOP are not well compared. The clarification of this problem would greatly benefit developing preventive and therapeutic strategies for PMOP. Our results confirm that both necroptosis and apoptosis contribute to the excessive loss of osteocytes and bone loss in OVX-induced osteoporosis in vivo and in vitro, and furthermore, necroptosis may produce a greater influence on the death of osteocytes than apoptosis at 8 weeks after OVX.

It is difficult to distinguish necroptotic cells and apoptotic cells due to the absence of specific cellular markers. We used several methods to determine necroptotic death of osteocytes in vivo and in vitro. TEM was used to illustrate the morphological and ultrastructure features of apoptotic and necrotic osteocytes (Galluzzi et al., 2009). The typical features of necroptotic cell osteocytes included the expansion of osteocyte volume, swelling of the organelles, rupture of the plasma membrane, loss of organelle contents, and extensive formation of intracellular vacuoles in the bone matrix of OVX rats, especially in the vehicle and zVAD groups. By contrast, only vehicle intervention was associated with cell shrinkage and nuclear condensation that were typical in apoptotic cells. Furthermore, treatment with $\mathrm{Nec}-1$ improved these ultra-microstructural changes. These findings confirm that necroptotic cell death was crucial for osteocyte loss. More interestingly, significantly fewer apoptotic osteocytes were observed by TEM than necrotic osteocytes. These indicated that necroptosis may produce a greater impact on the death of osteocytes than apoptosis in OVX-induced osteoporosis. The osteocytes are surrounded by the mineralized matrix and the apoptotic osteocytes are not rapidly engulfed by neighboring cells such as osteoclasts and macrophages (Cerri et al., 2000; Boabaid et al., 2001; FlorencioSilva et al., 2015), which indicates that the death of osteocytes is mainly caused by secondary necrosis and this supports our finding.

The TUNEL method was regarded as the gold standard to identify cell death, including necroptosis and apoptosis, both of which could generate DNA fragments and react with TUNEL staining mixtures (Trichonas et al., 2010). We used the TUNEL assay to detect dying cells in the bone matrix of OVX-induced rats. The results demonstrated that the number of TUNEL-positive cells and their percentage in the vehicle group were both increased. In addition, treatment with $\mathrm{Nec}-1$ significantly reduced the number and percentage of TUNELpositive cells than did zVAD. To further discriminate the types of TUNEL-positive cells observed, anti-active caspase-3 immunostaining was performed. Typically, TUNEL-positive cells and no immunoreactivity to the active caspase- 3 can be considered as necroptotic cells, while TUNEL-positive cells that exhibited positivity to the active caspase- 3 are characterized as apoptotic cells (Günther et al., 2011; Liedtke et al., 2011; Vanlangenakker et al., 2012). These methods also confirmed that these two types of cells were both significantly reduced in $\mathrm{Nec}-1$ and zVAD intervention, but $\mathrm{Nec}-1$-induced reduction appeared to be more obvious than $\mathrm{zVAD}$-induced reduction.

To obtain more convincing evidence, we cultured MLO-Y4 cells in vitro, and cell death was observed by TEM and quantitatively analyzed. The results showed that the incidence of apoptosis and necroptosis was significantly elevated in MLOY4 cells stimulated with TNF- $\alpha$. Importantly, we found that the incidence of necroptotic MLO-Y4 cells was significantly higher than that of apoptotic cells. These in vitro results were consistent with the results of OVX-induced rats. These results demonstrated that necroptosis had greater roles in osteocyte death than did apoptosis. However, these findings are not supported in the microarchitectural parameters, suggesting that apoptosis and necroptosis of osteocytes is just one important factor to affect bone loss and osteoporosis. Other factors including osteoclastic resorption and bone formation by osteogenic differentiation of the bone marrow mesenchymal stem cells also had valuable roles in bone loss and osteoporosis (Li et al., 2019; Wang et al., 2020; Zheng et al., 2021).

RIPK3 is a key switch molecule between the necroptotic and apoptotic pathways and is also recruited to the necrosome complex through direct interactions between RIP homotypic interaction motif domains of RIPK1 and RIPK3 (Galluzzi et al., 2009; Günther 
et al., 2011). (Linkermann et al., 2012) reported its functional predominance of necroptosis over apoptosis in renal ischemic/ reperfusion injury. Our study demonstrated that the protein level of RIPK3 was remarkably increased in the bone matrix of OVX rats, and this increase could be significantly blocked by Nec-1 but not $\mathrm{zVAD}$, suggesting that RIPK3 mainly regulated necroptotic cell death rather than apoptotic death. Additionally, if apoptotic osteocytes are not immediately cleared by macrophages, secondary necrosis occurs with cytoplasmic membrane ruptures. These stimulate the generation of multiple immunostimulatory cytokines and proinflammatory molecules, the aggregation and energization of immune cells (e.g., macrophages, monocytes, and neutrophils), which also induce the secretion of RANKL and bone resorption (Komori, 2013; Komori, 2016).

We also should consider several limitations. Firstly, we only used the bone matrix of the tibial plateaus after flushing out the medullary cavity during the extraction of protein. The harvested tibial plateaus used for western blotting not only contained mainly osteoblasts but also comprised other cells such as osteoclasts, which may affect the results of western blotting. Secondly, our results found that necroptosis may have a greater influence on the death of osteocytes than apoptosis at 8 weeks after OVX surgery, but only one follow-up time was included in our study. Necroptosis and apoptosis displayed different roles in the death of osteocytes at various time points. For instance, apoptosis appeared to be the most prevalent type of cell death in osteocytes within 14 days after OVX surgery, while the necroptosis had improved function to cause osteocyte death in the following weeks (Emerton et al., 2010; Cui et al., 2016b). Thirdly, necroptosis showed greater roles in osteocyte death than apoptosis at 8 weeks after OVX surgery, which was not consistent with the microarchitectural parameters of micro-CT in OVX rats, indicating that other factors such as osteoclastic resorption and osteogenic differentiation also affected bone mass and osteoporosis.

\section{REFERENCES}

Baumann, M., Janssen, B. J. A., Rob Hermans, J. J., Bartholome, R., Smits, J. F. M., and StruijkerBoudier, H. A. J. (2009). Renal Medullary Effects of Transient Prehypertensive Treatment in Young Spontaneously Hypertensive Rats. Acta Physiol. (Oxford, England) 196 (2), 231-237. doi:10.1111/j.1748-1716.2008.01916.x

Belizário, J., Vieira-Cordeiro, L., and Enns, S. (2015). Necroptotic Cell Death Signaling and Execution Pathway: Lessons from Knockout Mice. Mediators Inflamm. 2015, 128076. doi:10.1155/2015/128076

Boabaid, F., Cerri, P. S., and Katchburian, E. (2001). Apoptotic Bone Cells May Be Engulfed by Osteoclasts during Alveolar Bone Resorption in Young Rats. Tissue and Cell 33 (4), 318-325. doi:10.1054/tice.2001.0179

Cardoso, L., Herman, B. C., Verborgt, O., Laudier, D., Majeska, R. J., and Schaffler, M. B. (2009). Osteocyte Apoptosis Controls Activation of Intracortical Resorption in Response to Bone Fatigue. J. Bone Mineral Res. 24 (4), 597-605. doi:10.1359/jbmr.081210

Cerri, P. S., Freymüller, E., and Katchburian, E. (2000). Apoptosis in the Early Developing Periodontium of Rat Molars. Anat. Rec. 258 (2), 136-144. doi:10.1002/(sici)1097-0185(20000201)258:2<136:aid-ar3>3.0.co;2-1

Cui, H., Zhu, Y., and Jiang, D. (2016). The RIP1-RIP3 Complex Mediates Osteocyte Necroptosis after Ovariectomy in Rats. PloS one 11 (3), e0150805. doi:10.1371/ journal.pone.0150805

\section{CONCLUSION}

Both necroptosis and apoptosis are confirmed to result in the death and excessive loss of osteocytes in OVX-induced osteoporosis, and necroptosis may generate a greater impact on the loss of osteocytes than apoptosis.

\section{DATA AVAILABILITY STATEMENT}

The original contributions presented in the study are included in the article/Supplementary Material; further inquiries can be directed to the corresponding author.

\section{ETHICS STATEMENT}

The animal study was reviewed and approved by the Ethics Committee of the First Affiliated Hospital of Hainan Medical University.

\section{AUTHOR CONTRIBUTIONS}

$\mathrm{HC}, \mathrm{YZ}$, and $\mathrm{BH}$ conducted the study design. $\mathrm{BH}, \mathrm{HC}, \mathrm{YZ}, \mathrm{BS}, \mathrm{TS}$, and $\mathrm{PW}$ performed the experiments. $\mathrm{ZJ}, \mathrm{BH}, \mathrm{HC}$, and $\mathrm{BS}$ conducted the statistical analysis, data interpretation, manuscript preparation, and literature search. HC conducted funds collection.

\section{FUNDING}

This study was supported by grants from the National Natural Science Foundation of China (81760260) and Hainan Natural Science Foundation of Hainan Province (817326).

Cui, H., Zhu, Y., Yang, Q., Zhao, W., Zhang, S., Zhou, A., et al. (2016). Necrostatin1 Treatment Inhibits Osteocyte Necroptosis and Trabecular Deterioration in Ovariectomized Rats. Sci. Rep. 6, 33803. doi:10.1038/srep33803

Dallas, S. L., Prideaux, M., and Bonewald, L. F. (2013). The Osteocyte: An Endocrine Cell ... and Moreand More. Endocr. Rev. 34 (5), 658-690. doi:10.1210/er.2012-1026

Degterev, A., Zhou, W., Maki, J. L., and Yuan, J. (2014). Assays for Necroptosis and Activity of RIP Kinases. Methods Enzymol. 545, 1-33. doi:10.1016/b978-0-12801430-1.00001-9

Emerton, K. B., Hu, B., Woo, A. A., Sinofsky, A., Hernandez, C., Majeska, R. J., et al. (2010). Osteocyte Apoptosis and Control of Bone Resorption Following Ovariectomy in Mice. Bone 46 (3), 577-583. doi:10.1016/j.bone.2009.11.006

Ensrud, K. E., and Crandall, C. J. (2017). Osteoporosis. Ann. Intern. Med. 167 (3), Itc17-itc32. doi:10.7326/aitc201708010

Fang, H., Zhang, H., Wang, Z., Zhou, Z., Li, Y., and Lu, L. (2020). Systemic Immune-Inflammation index Acts as a Novel Diagnostic Biomarker for Postmenopausal Osteoporosis and Could Predict the Risk of Osteoporotic Fracture. J. Clin. Lab. Anal. 34 (1), e23016. doi:10.1002/jcla.23016

Florencio-Silva, R., Sasso, G. R., Sasso-Cerri, E., Simões, M. J., and Cerri, P. S. (2015). Biology of Bone Tissue: Structure, Function, and Factors that Influence Bone Cells. Biomed. Res. Int. 2015, 421746. doi:10.1155/2015/421746

Fu, R., Lv, W.-C., Xu, Y., Gong, M.-Y., Chen, X.-J., Jiang, N., et al. (2020). Endothelial ZEB1 Promotes Angiogenesis-dependent Bone Formation and Reverses Osteoporosis. Nat. Commun. 11 (1), 460. doi:10.1038/s41467-019-14076-3 
Galluzzi, L., Aaronson, S. A., Abrams, J., Alnemri, E. S., Andrews, D. W., Baehrecke, E. H., et al. (2009). Guidelines for the Use and Interpretation of Assays for Monitoring Cell Death in Higher Eukaryotes. Cell Death Differ 16 (8), 1093-1107. doi:10.1038/cdd.2009.44

Gerbaix, M., Gnyubkin, V., Farlay, D., Olivier, C., Ammann, P., Courbon, G., et al. (2017). One-month Spaceflight Compromises the Bone Microstructure, TissueLevel Mechanical Properties, Osteocyte Survival and Lacunae Volume in Mature Mice Skeletons. Sci. Rep. 7 (1), 2659. doi:10.1038/s41598-017-03014-2

Günther, C., Martini, E., Wittkopf, N., Amann, K., Weigmann, B., Neumann, H., et al. (2011). Caspase-8 Regulates TNF- $\alpha$-Induced Epithelial Necroptosis and Terminal Ileitis. Nature 477 (7364), 335-339. doi:10.1038/nature10400

Han, Y., You, X., Xing, W., Zhang, Z., and Zou, W. (2018). Paracrine and Endocrine Actions of Bone-The Functions of Secretory Proteins from Osteoblasts, Osteocytes, and Osteoclasts. Bone Res. 6, 16. doi:10.1038/s41413-018-0019-6

He, B., Yin, L., Zhang, M., Lyu, Q., Quan, Z., and Ou, Y. (2021). Causal Effect of Blood Pressure on Bone Mineral Density and Fracture: A Mendelian Randomization Study. Front. Endocrinol. 12 (910). doi:10.3389/fendo.2021.716681

Heino, T. J., Hentunen, T. A., and Väänänen, H. K. (2004). Conditioned Medium from Osteocytes Stimulates the Proliferation of Bone Marrow Mesenchymal Stem Cells and Their Differentiation into Osteoblasts. Exp. Cel. Res. 294 (2), 458-468. doi:10.1016/j.yexcr.2003.11.016

Kalajzic, I., Matthews, B. G., Torreggiani, E., Harris, M. A., Divieti Pajevic, P., and Harris, S. E. (2013). In Vitro and In Vivo Approaches to Study Osteocyte Biology. Bone 54 (2), 296-306. doi:10.1016/j.bone.2012.09.040

Kanis, J. A., Cooper, C., Cooper, C., Rizzoli, R., and Reginster, J.-Y. (2019). European Guidance for the Diagnosis and Management of Osteoporosis in Postmenopausal Women. Osteoporos. Int. 30 (1), 3-44. doi:10.1007/s00198018-4704-5

Kitaura, H., Marahleh, A., Ohori, F., Noguchi, T., Shen, W. R., Qi, J., et al. (2020). Osteocyte-Related Cytokines Regulate Osteoclast Formation and Bone Resorption. Int. J. Mol. Sci. 21 (14). doi:10.3390/ijms21145169

Komori, T. (2016). Cell Death in Chondrocytes, Osteoblasts, and Osteocytes. Int. J. Mol. Sci. 17 (12). doi:10.3390/ijms17122045

Komori, T. (2013). Functions of the Osteocyte Network in the Regulation of Bone Mass. Cell Tissue Res 352 (2), 191-198. doi:10.1007/s00441-012-1546-x

Li, J., Wang, P., Xie, Z., Wang, S., Cen, S., Li, M., et al. (2019). TRAF4 Positively Regulates the Osteogenic Differentiation of Mesenchymal Stem Cells by Acting as an E3 Ubiquitin Ligase to Degrade Smurf2. Cell Death Differ 26 (12), 2652-2666. doi:10.1038/s41418-019-0328-3

Liedtke, C., Bangen, J. M., Freimuth, J., Beraza, N., Lambertz, D., Cubero, F. J., et al. (2011). Loss of Caspase-8 Protects Mice against Inflammation-Related Hepatocarcinogenesis but Induces Non-apoptotic Liver Injury. Gastroenterology 141 (6), 2176-2187. doi:10.1053/j.gastro.2011.08.037

Linkermann, A., Bräsen, J. H., Himmerkus, N., Liu, S., Huber, T. B., Kunzendorf, U., et al. (2012). Rip1 (Receptor-interacting Protein Kinase 1) Mediates Necroptosis and Contributes to Renal Ischemia/reperfusion Injury. Kidney Int. 81 (8), 751-761. doi:10.1038/ki.2011.450

Liu, Z., Li, C., Huang, P., Hu, F., Jiang, M., Xu, X., et al. (2020). CircHmbox1 Targeting miRNA-1247-5p Is Involved in the Regulation of Bone Metabolism by TNF- $\alpha$ in Postmenopausal Osteoporosis. Front. Cell Dev. Biol. 8, 594785. doi:10.3389/fcell.2020.594785

Lu, J., Zhou, Z., Ma, J., Lu, N., Lei, Z., Du, D., et al. (2020). Tumour Necrosis Factora Promotes BMHSC Differentiation by Increasing P2X7 Receptor in Oestrogen-deficient Osteoporosis. J. Cell. Mol. Med. 24 (24), 14316-14324. doi:10.1111/jcmm.16048

Malik, M. A., Lone, S. A., Wani, M. Y., Talukdar, M. I. A., Dar, O. A., Ahmad, A., et al. (2020). S-benzyldithiocarbazate Imine Coordinated Metal Complexes Kill Candida Albicans by Causing Cellular Apoptosis and Necrosis. Bioorg. Chem. 98, 103771. doi:10.1016/j.bioorg.2020.103771

Mattinzoli, D., Messa, P., Corbelli, A., Ikehata, M., Mondini, A., Zennaro, C., et al. (2014). Application of Retinoic Acid to Obtain Osteocytes Cultures from Primary Mouse Osteoblasts. J. Vis. Exp. (87), 51465. doi:10.3791/51465

Milovanovic, P., Zimmermann, E. A., Hahn, M., Djonic, D., Püschel, K., Djuric, M., et al. (2013). Osteocytic Canalicular Networks: Morphological Implications for
Altered Mechanosensitivity. ACS nano 7 (9), 7542-7551. doi:10.1021/ nn401360u

Ormsby, R. T., Solomon, L. B., Yang, D., Crotti, T. N., Haynes, D. R., Findlay, D. M., et al. (2019). Osteocytes Respond to Particles of Clinically-Relevant Conventional and Cross-Linked Polyethylene and Metal Alloys by UpRegulation of Resorptive and Inflammatory Pathways. Acta Biomater. 87, 296-306. doi:10.1016/j.actbio.2019.01.047

Robinson, N., Ganesan, R., Hegedűs, C., Kovács, K., Kufer, T. A., and Virág, L. (2019). Programmed Necrotic Cell Death of Macrophages: Focus on Pyroptosis, Necroptosis, and Parthanatos. Redox Biol. 26, 101239. doi:10.1016/ j.redox.2019.101239

Robling, A. G., and Bonewald, L. F. (2020). The Osteocyte: New Insights. Annu. Rev. Physiol. 82, 485-506. doi:10.1146/annurev-physiol-021119-034332

$\mathrm{Ru}$, J.-y., and Wang, Y.-f. (2020). Osteocyte Apoptosis: the Roles and Key Molecular Mechanisms in Resorption-Related Bone Diseases. Cell Death Dis 11 (10), 846. doi:10.1038/s41419-020-03059-8

Tomkinson, A., Gevers, E. F., Wit, J. M., Reeve, J., and Noble, B. S. (1998). The Role of Estrogen in the Control of Rat Osteocyte Apoptosis. J. Bone Miner Res. 13 (8), 1243-1250. doi:10.1359/jbmr.1998.13.8.1243

Trichonas, G., Murakami, Y., Thanos, A., Morizane, Y., Kayama, M., Debouck, C. M., et al. (2010). Receptor Interacting Protein Kinases Mediate Retinal DetachmentInduced Photoreceptor Necrosis and Compensate for Inhibition of Apoptosis. Proc. Natl. Acad. Sci. 107 (50), 21695-21700. doi:10.1073/pnas.1009179107

Udagawa, N., Koide, M., Nakamura, M., Nakamichi, Y., Yamashita, T., Uehara, S., et al. (2021). Osteoclast Differentiation by RANKL and OPG Signaling Pathways. J. Bone Miner Metab. 39 (1), 19-26. doi:10.1007/s00774-020-01162-6

Vakili, S., Zal, F., Mostafavi-pour, Z., Savardashtaki, A., and Koohpeyma, F. (2021). Quercetin and Vitamin E Alleviate Ovariectomy-induced Osteoporosis by Modulating Autophagy and Apoptosis in Rat Bone Cells. J. Cell Physiol 236 (5), 3495-3509. doi:10.1002/jcp.30087

Vanlangenakker, N., Vanden Berghe, T., and Vandenabeele, P. (2012). Many Stimuli Pull the Necrotic Trigger, an Overview. Cell Death Differ 19 (1), 75-86. doi:10.1038/cdd.2011.164

Wang, L., You, X., Lotinun, S., Zhang, L., Wu, N., and Zou, W. (2020). Mechanical Sensing Protein PIEZO1 Regulates Bone Homeostasis via Osteoblast-Osteoclast Crosstalk. Nat. Commun. 11 (1), 282. doi:10.1038/s41467-019-14146-6

Zhao, J., Zhang, M., Quan, Z., Deng, L., Li, Y., and He, B. (2021). Systematic Influence of Circulating Bilirubin Levels on Osteoporosis. Front. Endocrinol. 12 (1022). doi:10.3389/fendo.2021.719920

Zheng, Z., Zhang, X., Huang, B., Liu, J., Wei, X., Shan, Z., et al. (2021). Site-1 Protease Controls Osteoclastogenesis by Mediating LC3 Transcription. Cell Death Differ. 28 (6), 2001-2018. doi:10.1038/s41418-020-00731-6

Zhu, Y., Cui, H., Xia, Y., and Gan, H. (2016). RIPK3-Mediated Necroptosis and Apoptosis Contributes to Renal Tubular Cell Progressive Loss and Chronic Kidney Disease Progression in Rats. PloS one 11 (6), e0156729. doi:10.1371/ journal.pone.0156729

Conflict of Interest: The authors declare that the research was conducted in the absence of any commercial or financial relationships that could be construed as a potential conflict of interest.

Publisher's Note: All claims expressed in this article are solely those of the authors and do not necessarily represent those of their affiliated organizations, or those of the publisher, the editors, and the reviewers. Any product that may be evaluated in this article, or claim that may be made by its manufacturer, is not guaranteed or endorsed by the publisher.

Copyright (c) $2021 \mathrm{He}, \mathrm{Zhu}, \mathrm{Cui}$, Sun, Su and Wen. This is an open-access article distributed under the terms of the Creative Commons Attribution License (CC BY). The use, distribution or reproduction in other forums is permitted, provided the original author(s) and the copyright owner(s) are credited and that the original publication in this journal is cited, in accordance with accepted academic practice. No use, distribution or reproduction is permitted which does not comply with these terms. 